\title{
Análise psicométrica dos itens da Avaliação da Coordenação \\ e Destreza Motora (ACOORDEM) em crianças de 4 anos
}

\section{Psychometric analysis of items of Assessment of Motor Coordination and Dexterity (Acoordem) for 4-year-old children}

\author{
Cynthia Girundi da Silva ${ }^{1}$, Adriana Maria Valladão Novais Van Petten ${ }^{2}$, \\ Estefânia Harsányi ${ }^{2}$, Lívia de Castro Magalhães ${ }^{2}$
}

http://dx.doi.org/10.11606/issn.2238-6149.v28i1p9-18

Silva CGS, Van Petten, AMVN, Harsányi E, Magalhaes LC. Análise psicométrica dos itens da Avaliação da Coordenação e Destreza Motora (ACOORDEM) em crianças de 4 anos. Rev Ter Ocup Univ São Paulo. 2017 jan.-abr.;28(1):9-18.

RESUMO: A Avaliação da Coordenação e Destreza Motora - ACOORDEM é um teste motor criado para crianças brasileiras de 4 a 8 anos. O objetivo desse estudo foi investigar a validade e confiabilidade da ACOORDEM para crianças de 4 anos. Foram avaliadas 80 crianças, divididas em grupos iguais por sexo e tipo de escola. Os pais e professores também participaram da pesquisa respondendo questionários. O reteste foi feito com dez participantes. Poucos itens apresentaram diferença estatisticamente significativa associada ao sexo e tipo de escola. A confiabilidade teste reteste apresentou bons índices. A ACOORDEM é adequada para avaliação motora de crianças de 4 anos, contudo alguns itens necessitam ser revisados para melhorar a confiabilidade do instrumento e reduzir o tempo de aplicação.

DESCRITORES: Transtornos das habilidades motoras; Criança; Pré-escolar; Reprodutibilidade dos testes; Avaliação.
Silva CGS, Van Petten, AMVN, Harsányi E, Magalhaes LC. Psychometric analysis of items of Assessment of Motor Coordination and Dexterity (ACOORDEM) for 4-year-old children. Rev Ter Ocup Univ São Paulo. 2017 Jan.-Apr.;28(1):9-18.

ABSTRACT: The Assessment of Motor Coordination and Dexterity - ACOORDEM is a standardized motor assessment for Brazilian children aged 4-8 years. This study aimed to investigate the validity and reliability of the test for 4-yearold children. Eighty children of 4 years old, divided into equal groups by sex and school type (public or private), were evaluated. Parents and teachers also answered the questionnaires. The retest was applied to 10 participants. Few items showed a statistically significant difference associated with sex and school type. The test-retest reliability presented good indexes. ACOORDEM is suitable for the motor assessment of 4-year-old children; however, some items need to be revised or discarded to improve the instrument reliability and reduce the administration time.

KEYWORDS: Motor skills disorders; Child; Child, preschool; Reproducibility of results; Evaluation.

Este artigo é parte da dissertação: de Cynthia Girundi da Silva "Análise psicométrica dos itens da Avaliação da Coordenação e Destreza Motora (ACOORDEM) em crianças de 4 anos", 2014. Apresentado na 11th International Conference on Developmental Coordination Disorder, Toulouse, França, 2015.

1. Curso de Terapia Ocupacional, Universidade Federal de Pelotas (UFPel), Pelotas, RS, Brasil.

2. Departamento de Terapia Ocupacional, Universidade Federal de Minas Gerais (UFMG), Belo Horizonte, MG, Brasil. Email: avalladao@gmail.com, esteharsanyi@hotmail.com, liviacmag@gmail.com Agências de fomento: Fapemig e CNPq.

Endereço para correspondência: Prof ${ }^{a}$ Cynthia Girundi da Silva. Departamento de Terapia Ocupacional, Universidade Federal de Pelotas. Av. Duque de Caxias, 250, Fragata - CEP: 96030-000, Pelotas, Rio Grande do Sul, Brasil. E-mail: cynthiagirundi@gmail.com 


\section{INTRODUÇÃ̃O}

A avaliação motora identifica alterações no desempenho motor, possibilitando a oferta de suporte adequado para as crianças e suas famílias a fim de reduzir o impacto de dificuldades motoras nas atividades diárias e na vida escolar. É importante que a detecção ocorra precocemente, de preferência antes do ingresso no ensino fundamental, para que os efeitos da dificuldade motora sobre o desempenho acadêmico e socialização sejam minimizados ${ }^{1}$. Por outro lado, quando são observadas alterações motoras em crianças préescolares não há consenso na literatura se o resultado da avaliação caracteriza um transtorno, como o Transtorno do Desenvolvimento da Coordenação (TDC), ou se é um atraso no desenvolvimento ocasionado pela falta de oportunidade para experimentar e praticar atividades motoras, que auxiliam no repertório motor e, consequentemente, dão suporte ao bom desempenho nas avaliações ${ }^{2}$.

Existem testes com boas qualidades psicométricas para avaliação do desempenho motor ${ }^{1,3}$ que apresentam características variadas, cobrindo o desenvolvimento das habilidades motoras do nascimento a adolescência. Contudo, todos são estrangeiros, sendo necessário traduzir, adaptar e investigar a validade e confiabilidade para crianças brasileiras. Apesar do Movement Assessment Battery for Chidren-2 (MABC-2) ter sido recentemente traduzido e ter apresentado boa confiabilidade com crianças brasileiras ${ }^{4}$, o teste ainda conta com empecilhos para seu uso clínico, como o alto custo de importação e a dificuldade de reposição de peças.

Ressalta-se que os principais testes de desempenho motor, não incluem atividades relevantes para o contexto escolar que exigem desempenho motor, como por exemplo, a escrita e a participação nas tarefas de sala de aula. Por isso, frequentemente o critério B do Diagnostic and Statistical Manual of Mental Disorders- 5 (DSM-5) $)^{5}$ para diagnóstico do TDC não é avaliado. O critério B especifica que esse diagnóstico só é pertinente se as dificuldades motoras (critério A), sem adaptação, têm impacto no desempenho acadêmico. Avaliar a escrita é especialmente importante, pois é uma das metas de intervenção mais frequentes para essas crianças ${ }^{6}$ já que a dificuldade de escrita manual pode prejudicar o desempenho acadêmico, com impacto na autoestima, nas relações pessoais e nas percepções da criança e dos outros acerca de suas habilidades ${ }^{7}$.

Face à dificuldade de acesso a testes importados e à ausência de avaliação motora padronizada para crianças brasileiras, principalmente testes que englobam todos os critérios diagnósticos para o TDC, foi criada a Avaliação da Coordenação e Destreza Manual (ACOORDEM) ${ }^{8}$. A ACOORDEM é um teste descritivo para diagnóstico motor de crianças de 4 a 8 anos, com a perspectiva de que também seja um instrumento avaliativo, para mensurar mudanças ao longo do tempo como resultado de intervenção ${ }^{9}$. O processo de criação do teste baseou-se na Classificação Internacional de Funcionalidade, Incapacidade e Saúde ${ }^{10,11}$ e estudos de validade e confiabilidade do instrumento para crianças de 6 a 8 anos, já foram realizados ${ }^{9,11,12,13}$.

A ACOORDEM é composta por itens para avaliar aspectos do desenvolvimento motor e funcional relevantes para a participação social e desempenho escolar. Além de itens motores e dos questionários para pais e professores, a ACOORDEM inclui duas provas de escrita - cópia do alfabeto e de sentença simples nas quais são avaliadas questões como velocidade e legibilidade, sendo também registrado o padrão de preensão no lápis, dentre os dez padrões descritos por Schneck e Henderson ${ }^{14}$. Embora não exista consenso na literatura acerca da influência dos diferentes padrões de preensão no lápis na qualidade da escrita ${ }^{15}$ observase maior frequência de padrões atípicos entre crianças com problemas de coordenação motora ${ }^{16}$. Assim, é importante registar os padrões de preensão no lápis observados em crianças brasileiras, pois isso pode contribuir para o diagnóstico motor.

$\mathrm{O}$ presente estudo dá continuidade ao processo de criação da ACOORDEM e tem como objetivos: (a) investigar a confiabilidade teste reteste do instrumento; (b) identificar fatores que podem influenciar a validade investigando se existem diferenças significativas no desempenho na ACOORDEM relacionadas ao sexo e tipo de escola; (c) estabelecer normas preliminares de desempenho motor para crianças brasileiras de 4 anos de idade e, (d) descrever os padrões de preensão no lápis durante as provas de escrita da ACOORDEM.

\section{MÉTODO}

\section{Participantes}

Participaram deste estudo 80 crianças de 4 anos de idade, recrutadas de forma aleatória em três escolas da rede pública (40 crianças) e sete da rede particular de Belo Horizonte (40 crianças), com igual representação de meninos e meninas. O cálculo amostral teve como base estudo anterior da ACOORDEM ${ }^{12}$ quando foi 
calculado que para um tamanho de efeito de 0,32 e poder estatístico de 0,90 , seria necessária amostra de 62 crianças por faixa etária.

Os critérios de inclusão foram: crianças com 4 anos, de ambos os sexos, nascidas a termo, cursando o ensino infantil, sem sinais de deficiência motora, visual, auditiva e/ou cognitiva, cujos pais ou responsáveis tivessem o ensino fundamental completo e os professores das crianças aceitassem participar do estudo. Foi permitida a inclusão de crianças que faziam uso de correção visual com óculos, porém não foram incluídas crianças que faziam uso de aparelho auditivo. Foram excluídas do estudo, crianças nascidas com idade gestacional inferior a 37 semanas e com peso ao nascimento inferior a 2500 gramas, que faziam algum tipo de terapia motora especializada, com história de fracasso escolar/repetência e que apresentassem sinais de déficit cognitivo.

Os pais ou responsáveis de todos os participantes, assim como os professores, assinaram o Termo de Consentimento Livre e Esclarecido (TCLE). Este estudo foi aprovado pelo Comitê de Ética em Pesquisa da UFMG (Parecer n ${ }^{\circ}$ ETIC 0647.0.203.000-10) em 23 de março de 2011.

\section{Instrumentos}

A ACOORDEM tem como objetivo detectar dificuldades de coordenação motora em crianças de 4 a 8 anos de idade. O teste é subdividido nas áreas de (1) Coordenação, Destreza Manual e Escrita (16 itens), (2) Coordenação Corporal e Planejamento Motor (26 itens) e (3) Atividades e Participação em Casa e na Escola (Questionário de pais com 54 itens e de professores com 30 itens), sendo que alguns itens observacionais são duplicados (ex. equilíbrio perna direita e esquerda), resultando em um total de 60 itens para as idades de 6 a 8 anos. Para adequar a complexidade do teste à idade algumas provas foram modificadas ou retiradas para crianças de 4 anos. As provas de escrita para pré-escolares foram adaptadas passando a constar de cópia de figuras geométricas simples e das vogais, ao invés da cópia do alfabeto e de uma sentença, como proposto para crianças de 6 a 8 anos. Assim, o teste para crianças de 4 anos totaliza em 51 itens. As áreas (1) e (2) são compostas por provas executadas pelas crianças considerando o tempo ou acuidade de execução da tarefa. Durante as provas de escrita foi registrado o tempo de execução e o padrão de preensão utilizado no lápis. Com relação aos questionários, o de pais é dividido em quatro subescalas: mobilidade, atividades de vida diária, papel de estudante e comportamento. O questionário de professores é dividido em duas subescalas: motora e comportamento. As respostas, em ambos os questionários, são pontuadas em escala de quatro pontos com registro de frequência, variando de 1 $=$ raro/nunca a $4=$ sempre .

Embora a ACOORDEM esteja em desenvolvimento, estudos anteriores reportam bons índices de confiabilidade para as idades de 6 a 8 anos ${ }^{9,11,12,13}$. O tempo médio para aplicação do teste completo com a criança é de aproximadamente 60 minutos.

A Escala de Maturidade Mental Colúmbia EMMC é um teste validado para crianças brasileiras ${ }^{17}$ que tem como objetivo fornecer estimativa da habilidade intelectual de crianças com idade mental de 3 anos e 11 meses a 9 anos e 11 meses. A capacidade de raciocínio é avaliada por meio de respostas não verbais, com uso de cartões com desenhos. Este teste foi usado para excluir crianças com sinais de deficiência intelectual.

\section{Procedimentos}

Para seleção da amostra foi realizado sorteio das crianças em cada turma nas escolas, seguido do envio dos envelopes aos pais contendo o Termo de Consentimento Livre e Esclarecido - TCLE, um breve questionário sobre o histórico do desenvolvimento da criança, os questionários de pais da ACOORDEM e o CCEB. Participaram do estudo as crianças que atendiam aos critérios de inclusão. As crianças foram avaliadas com o MABC-2, EMMC e ACOORDEM nas dependências de sua escola, em horários definidos pelas professoras. As crianças foram convidadas para a sala de avaliação em duplas, uma vez que esta estratégia aumentava a colaboração e permanência na sala de teste, mas a avaliação foi realizada individualmente. Durante as provas de escrita, as mãos das crianças foram fotografadas para registrar o tipo de padrão de preensão no lápis utilizado. As avaliações ocorreram em dois dias, com sessões de 40 a 60 minutos.

Antes do início da coleta de dados, as examinadoras foram treinadas no MABC-2 e ACOORDEM. Para investigar a confiabilidade teste reteste da ACOORDEM, foram selecionadas por conveniência 10 crianças, dentre as 80 que participaram do estudo, em diferentes escolas. Para seleção dessas crianças foram considerados a disponibilidade de tempo, o espaço das escolas e a possibilidade de estruturação do cronograma para que a reavaliação ocorresse dentro 
do período de 7 a 14 dias após a primeira avaliação. O coeficiente de correlação intraclasse (CCI), modelo 2.1, consistência, foi utilizado para medir a confiabilidade teste reteste, interpretado conforme CICCHETTI ${ }^{18}$ : correlação pobre $(<0,40)$, correlação moderada (entre 0,40 e 0,59 ), correlação boa (entre 0,60 e 0,74 ) e correlação excelente $(>0,75)$. Considerando, no entanto, a dificuldade de se obter concordância absoluta para as variáveis quantitativas medidas em termos de tempo em segundos e número de erros, o teste $t$-student pareado foi utilizado para avaliar a confiabilidade teste reteste dessas variáveis.

O programa estatístico SPSS, versão 17.0, foi usado para análise dos dados. A descrição da amostra quanto a idade, sexo, tipo de escola, classe econômica, a pontuação nos testes e padrão de preensão no lápis foi feita em termos de frequência, média e desvio padrão. ANOVA foi utilizada para comparação de variáveis quantitativas quanto ao sexo (feminino e masculino) e tipo de escola (pública e particular). Para comparação das variáveis qualitativas foi utilizado o teste de Mann-Whitney. Em todas as análises foi considerado nível de significância alpha de 0,05 .

\section{RESULTADOS}

Dos 145 envelopes distribuídos, 98 retornaram às pesquisadoras. Desses, 80 foram incluídos na amostra. Das 18 crianças excluídas, 16 pais não atendiam ao critério de escolaridade, uma criança tinha história de prematuridade e uma não obteve pontuação suficiente no EMMC.

As 80 crianças avaliadas tinham idade média de $54,0( \pm 0,7)$ meses e peso médio ao nascimento de 3,210 ( \pm 99$)$ gramas. Em relação à dominância, $88,8 \%$ eram destras. Com relação ao EMMC, não foi encontrada diferença estatisticamente significativa ao comparar o desempenho entre os sexos $(p=0,506)$, mas as crianças das escolas particulares apresentaram escore mais elevado do que as das escolas públicas $(\mathrm{p}=0,016)$.

Tabela 1 - Itens quantitativos e qualitativos da ACOORDEM

\begin{tabular}{|c|c|c|c|}
\hline \multicolumn{2}{|c|}{ Coordenação Manual e Destreza Motora } & \multicolumn{2}{|c|}{ Coordenação Bilateral e planejamento motor } \\
\hline Quantitativos & Qualitativos & Quantitativos & Qualitativos \\
\hline Colocar pinos tábua mão preferida $\S$ & Cópia de figuras- quadrado $\varnothing$ & Agarrar saco de areia $\varnothing$ & Batucada $1 \varnothing$ \\
\hline Colocar pinos tábua mão não preferida $\S$ & Cópia de figuras- $X \varnothing$ & Repica bola $20 \mathrm{~cm} \varnothing$ & Batucada $2 \varnothing$ \\
\hline Mudar pinos fileira preferida $\S$ & Cópia de figuras- peixe $\phi$ & Agarrar bola na parede $\varnothing$ & Batucada $3 \phi$ \\
\hline Mudar pinos fila não preferida $\S$ & $\begin{array}{l}\text { Cópia de figuras- } 3 \text { linhas } \\
\text { cruzadas } \varnothing\end{array}$ & Labirinto quadrado mão preferida $\S$ & Batucada $4 \varnothing$ \\
\hline Pesponto $\S$ & $\begin{array}{l}\text { Cópia de figuras- círculo/ } \\
\text { quadrado aberto } \varnothing\end{array}$ & Labirinto quadrado mão não preferida $\S$ & Amarelinha 2 pés laterais $\varnothing$ \\
\hline Colocar moedas cofre mão preferida $\S$ & Cópia de figuras- 3 círculos $\phi$ & Labirinto árvore mão preferida $\S$ & Amarelinha $1221 \varnothing$ \\
\hline Colocar moedas cofre mão não preferida $\S$ & $\begin{array}{l}\text { Cópia de figuras- círculo e } \\
\text { triângulo } \varnothing\end{array}$ & Labirinto árvore mão não preferida $\S$ & Amarelinha $12112 \varnothing$ \\
\hline Distribuir cartas $\S$ & $\begin{array}{l}\text { Cópia de figuras- losango } \\
\text { horizontal } \varnothing\end{array}$ & Prono extensão tempo $£$ & Polichinelo $\varnothing$ \\
\hline Polegar dedos mão preferida $\S$ & & Supino flexão tempo $£$ & Prono extensão acuidade $\varnothing$ \\
\hline Polegar dedos mão não preferida $\S$ & & Equilíbrio OAD $£$ & Supino flexão acuidade $\varnothing$ \\
\hline Traçado reto \# & & Equilíbrio OAE $£$ & \\
\hline Traçado curvo \# & & Equilíbrio OFD $£$ & \\
\hline Cópia de figuras escore total $\varnothing$ & & Equilíbrio OFE $£$ & \\
\hline Cópia de símbolo $\S$ & & Circuito tempo total $\S$ & \\
\hline Cópia de vogais $\S$ & & Tandem número passos $\varnothing$ & \\
\hline Recorte reto 4anos \# & & Coelho número pulos $\varnothing$ & \\
\hline Recorte curvo 4anos \# & & & \\
\hline
\end{tabular}


Silva CG, et al. Análise psicométrica dos itens da ACOORDEM. Rev Ter Ocup Univ São Paulo. 2017 jan./abr.;28(1):9-18.

Tabela 2 - Média e comparação dos itens quantitativos da ACOORDEM - Coordenação e destreza manual - por sexo e tipo de escola

\begin{tabular}{|c|c|c|c|c|c|c|}
\hline & Feminino & Masculino & & Particular & Pública & \\
\hline & $\begin{array}{c}\text { Média } \\
\text { (Desvio } \\
\text { Padrão) }\end{array}$ & $\begin{array}{c}\text { Média } \\
\text { (Desvio } \\
\text { Padrão) }\end{array}$ & $\begin{array}{c}\text { Valor } \\
\text { p }\end{array}$ & $\begin{array}{c}\text { Média } \\
\text { (Desvio } \\
\text { Padrão) }\end{array}$ & $\begin{array}{c}\text { Média } \\
\text { (Desvio } \\
\text { Padrão) }\end{array}$ & $\begin{array}{l}\text { Valor } \\
\text { p }\end{array}$ \\
\hline $\begin{array}{l}\text { Colocar pinos tábua mão } \\
\text { preferida } \S\end{array}$ & $22,6(5,00)$ & $23,3(6,5)$ & 0,604 & $23,1(6,60)$ & $22,8(4,80)$ & 0,839 \\
\hline $\begin{array}{l}\text { Colocar pinos tábua mão não } \\
\text { preferida } \S\end{array}$ & $27,4(7,00)$ & $28,8(7,20)$ & 0,405 & $26,8(6,60)$ & $29,4(7,50)$ & 0,136 \\
\hline Mudar pinos fileira preferida $\S$ & $28,8(6,20)$ & $32,4(8,20)$ & 0,054 & $30,0(7,70)$ & $31,4(7,20)$ & 0,457 \\
\hline Mudar pinos fila não preferida $\S$ & $33,1(6,00)$ & $36,2(7,30)$ & 0,112 & $34,6(7,40)$ & $34,7(6,00)$ & 0,961 \\
\hline Pesponto $\S$ & $52,7(26,0)$ & $46,3(18.60)$ & 0,242 & $45,6(20,60)$ & $53,2(24,20)$ & 0,168 \\
\hline $\begin{array}{l}\text { Colocar moedas cofre mão } \\
\text { preferida } \S\end{array}$ & $9,55(3,17)$ & $9,31(3,51)$ & 0,749 & $8,89(2,48)$ & $9,93(3,92)$ & 0,175 \\
\hline $\begin{array}{l}\text { Colocar moedas cofre mão não } \\
\text { preferida } \S\end{array}$ & $11,0(4,20)$ & $9,6(2,60)$ & 0,094 & $10,0(2,50)$ & $10,6(4,30)$ & 0,511 \\
\hline Distribuir cartas $\S$ & $24,4(7,70)$ & $31,6(11,60)$ & 0,002 & $27,8(9,70)$ & $27,9(11,1)$ & 0,975 \\
\hline Polegar dedos mão preferida $\S$ & $17,1(6,70)$ & $21(10,20)$ & 0,064 & $20,5(10,20)$ & $17,4(6,70)$ & 0,154 \\
\hline $\begin{array}{l}\text { Polegar dedos mão não } \\
\text { preferida } \S\end{array}$ & $19,5(9,80)$ & $22,3(12,40)$ & 0,308 & $21,8(12,30)$ & $20,0(10,10)$ & 0,507 \\
\hline Traçado reto \# & $4,3(5,23)$ & $5,25(6,77)$ & 0,485 & $4,63(6,40)$ & $4,93(5,72)$ & 0,826 \\
\hline Traçado curvo \# & $16,3(12,30)$ & $17,3(11,50)$ & 0,695 & $16,1(10,3)$ & $17,6(13,3)$ & 0,576 \\
\hline Cópia de figuras escore total $\varnothing$ & $1,55(1,26)$ & $1,53(1,15)$ & 0,927 & $1,8(1,34)$ & $1,28(0,99)$ & 0,05 \\
\hline Cópia de símbolo $\S$ & $18,7(7,10)$ & $23,8(9,40)$ & 0,009 & $20,3(8,90)$ & $22,2(8,40)$ & 0,349 \\
\hline Cópia de vogais $\S$ & $20,7(5,70)$ & $24,4(12,0)$ & 0,083 & $21,8(8,70)$ & $23,3(10,40)$ & 0,478 \\
\hline Recorte reto 4anos \# & $1,54(2,34)$ & $1,59(2,07)$ & 0,921 & $1,49(1,80)$ & $1,66(2,57)$ & 0,744 \\
\hline Recorte curvo 4anos \# & $4,77(4,23)$ & $6,94(6,37)$ & 0,096 & $4,22(3,59)$ & $7,57(6,56)$ & 0,009 \\
\hline
\end{tabular}

Nota: $\S=$ tempo em segundos: menor escore, melhor desempenho; \# = número de erros: menor o escore, melhor desempenho; $\not=$ número de acertos: maior escore, melhor desempenho. Números em negrito indicam diferença significativa.

Fonte: dados da pesquisa

Os resultados comparativos da ACOORDEM em relação ao sexo e tipo de escola estão especificados por subáreas do teste. A Tabela 1 apresenta as variáveis quantitativas e qualitativas. As Tabelas 2 e 3 mostram, respectivamente, a comparação dos itens quantitativos da ACOORDEM na subárea de coordenação destreza manual; e na subárea de coordenação bilateral e planejamento motor. Os itens qualitativos da ACOORDEM estão especificados na Tabela 4.

Ao comparar o desempenho em relação ao sexo, somente três itens $(5,9 \%)$ (destacados nas Tabelas $2 \mathrm{e}$
4) dos 51 aplicados na idade de 4 anos apresentaram diferença estatisticamente significativa, sendo que as meninas obtiveram melhor desempenho nos três itens.

Comparando o desempenho em relação ao tipo de escola, foi observada diferença estatisticamente significativa em sete itens $(13,7 \%)$ (destacados nas Tabelas 2 e 4). Nesses itens as crianças de escola particular apresentaram melhor desempenho do que as de escola pública. Pode-se observar, nas Tabelas 2 a 4, que alguns itens receberam pontuação mínima, indicando que foram muito difíceis para a amostra. 
Silva CG, et al. Análise psicométrica dos itens da ACOORDEM. Rev Ter Ocup Univ São Paulo. 2017 jan./abr.;28(1):9-18.

Tabela 3 - Média e comparação dos itens quantitativos da ACOORDEM - Coordenação bilateral e planejamento motor - por sexo e tipo de escola

\begin{tabular}{|c|c|c|c|c|c|c|}
\hline \multirow{3}{*}{$\begin{array}{l}\text { Itens quantitativos } \\
\text { da ACOORDEM }\end{array}$} & \multicolumn{2}{|c|}{ Sexo } & \multicolumn{4}{|c|}{ Tipo de escola } \\
\hline & Feminino & Masculino & \multirow[b]{2}{*}{$\begin{array}{c}\text { Valor } \\
\mathbf{p}\end{array}$} & \multirow{2}{*}{$\begin{array}{c}\text { Particular } \\
\text { Média (Desvio } \\
\text { Padrão) }\end{array}$} & \multirow{2}{*}{$\begin{array}{c}\text { Pública } \\
\begin{array}{c}\text { Média (Desvio } \\
\text { Padrão) }\end{array}\end{array}$} & \multirow[b]{2}{*}{$\begin{array}{c}\text { Valor } \\
\mathbf{p}\end{array}$} \\
\hline & $\begin{array}{l}\text { Média (Desvio } \\
\text { Padrão) }\end{array}$ & $\begin{array}{l}\text { Média (Desvio } \\
\text { Padrão) }\end{array}$ & & & & \\
\hline Agarrar saco de areia $\varnothing$ & $2,30(1,40)$ & $2,45(1,45)$ & 0,639 & $2,33(1,38)$ & $2,43(1,47)$ & 0,755 \\
\hline Repica bola $20 \mathrm{~cm} \varnothing$ & $1,83(1,69)$ & $2,08(1,82)$ & 0,526 & $1,93(1,79)$ & $1,98(1,73)$ & 0,899 \\
\hline Agarrar bola na parede $\varnothing$ & $0,03(0,16)$ & $0,10(0,44)$ & 0,315 & $0,05(0,32)$ & $0,08(0,35)$ & 0,738 \\
\hline $\begin{array}{l}\text { Labirinto quadrado mão } \\
\text { preferida } \S\end{array}$ & $5,73(2,08)$ & $6,76(4,44)$ & 0,207 & $6,34(3,97)$ & $6,14(2,94)$ & 0,804 \\
\hline $\begin{array}{l}\text { Labirinto quadrado mão não } \\
\text { preferida } \S\end{array}$ & $5,73(2,38)$ & $5,86(3,73)$ & 0,853 & $5,61(2,71)$ & $6,0(3,51)$ & 0,588 \\
\hline $\begin{array}{l}\text { Labirinto árvore mão } \\
\text { preferida } \S\end{array}$ & $14,2(5,40)$ & $13,4(4,80)$ & 0,56 & $13,8(4,80)$ & $13,8(5,40)$ & 0,299 \\
\hline $\begin{array}{l}\text { Labirinto árvore mão não } \\
\text { preferida } \S\end{array}$ & $15,1(7,80)$ & $14(5,30)$ & 0,532 & $15,5(8,10)$ & $13,6(4,60)$ & 0,248 \\
\hline Prono extensão tempo $£$ & $14,0(9,00)$ & $11,1(8,10)$ & 0,142 & $12,2(8,90)$ & $12,9(8,50)$ & 0,744 \\
\hline Supino flexão tempo $£$ & $10,28(7,60)$ & $8,0(6,44)$ & 0,171 & $9,32(7,75)$ & $8,92(6,42)$ & 0,808 \\
\hline Equilíbrio OAD $£$ & $8,08(6,04)$ & $6,43(5,89)$ & 0,22 & $6,3(4,70)$ & $8,2(6,97)$ & 0,157 \\
\hline Equilíbrio OAE $£$ & $5,43(3,85)$ & $6,43(5,95)$ & 0,375 & $5,58(4,60)$ & $6,28(5,42)$ & 0,535 \\
\hline Equilíbrio OFD $£$ & $2,4(1,03)$ & $2,2(1,26)$ & 0,441 & $2,15(0,83)$ & $2,45(1,40)$ & 0,247 \\
\hline Equilíbrio OFE $£$ & $2,69(2,07)$ & $2,03(1,22)$ & 0,087 & $2,47(1,97)$ & $2,25(1,46)$ & 0,569 \\
\hline Circuito tempo total $\S$ & $29,1(7,90)$ & $27,4(10,3)$ & 0,394 & $28,5(8,20)$ & $28,0(10,0)$ & 0,789 \\
\hline $\begin{array}{l}\text { Marcha Tandem - número } \\
\text { passos } \varnothing\end{array}$ & $3,4(3,69)$ & $2,3(2,29)$ & 0,113 & $2,98(2,89)$ & $2,73(3,34)$ & 0,721 \\
\hline Coelho número pulos $\varnothing$ & $4,18(1,15)$ & $3,68(1,47)$ & 0,095 & $3,98(1,49)$ & $3,88(1,18)$ & 0,741 \\
\hline
\end{tabular}

Nota: $\S=$ tempo em segundos: menor escore, melhor desempenho; $£=$ tempo em segundos: maior escore, melhor desempenho; \# = nú mero de erros: menor o escore, melhor desempenho; $\varnothing$ = número de acertos: maior escore, melhor desempenho. Números em negrito indicam diferença significativa. Fonte: dados da pesquisa

Em relação à confiabilidade teste reteste dos itens quantitativos, a pontuação de $10(30,3 \%)$ dos 33 itens - "polegar dedos mão preferida" ( $(\mathrm{p}=0,008)$, "polegar dedos mão não preferida" ( $\mathrm{p}=0,001)$, "recorte reto" ( $\mathrm{p}=0,049)$, "agarra bola tênis mão preferida" ( $\mathrm{p}=$ $0,001)$, "labirinto de estrela quadrado mão preferida" $(p=0,009)$, "labirinto de estrela quadrado mão não preferida" $(\mathrm{p}=0,003)$, "labirinto de complexo árvore mão preferida" $(\mathrm{p}=0,001)$, "labirinto de complexo árvore mão não preferida" $(\mathrm{p}=0,046)$, "circuito tempo total" $(\mathrm{p}=0,005)$, "coelho número pulos" $(\mathrm{p}=$ $0,001)$ - apresentou diferença média estatisticamente significante para aplicação nas duas ocasiões. Os demais itens apresentaram médias similares no teste reteste. Já nos itens qualitativos, o CCI identificou que 17 (94,4 $\%)$ dos 18 itens apresentam confiabilidade moderada a excelente com índices variando de 0,47 a 0,98 . Somente um item "batucada 1 " $(p=0,102)$ apresentou confiabilidade pobre.

Com relação aos padrões de preensão no lápis, dos dez padrões descritos na literatura ${ }^{16}$, seis foram identificados nesta amostra: padrão palmar supinado $1,3 \%$, polegar cruzado $1,3 \%$, trípode estática em $3,8 \%$, 4 dedos $28,8 \%$, trípode lateral $27,5 \%$, trípode dinâmica $21,3 \%$ e outros tipos $16 \%$. Ao analisar o tipo de escola foi identificado que $45 \%$ das crianças das escolas públicas adquiriram padrão de preensão maduro, ou seja trípode lateral ou trípode dinâmica, em comparação aos 52,5\% das crianças das escolas particulares. Em relação ao sexo, foi identificado que $55 \%$ dos meninos apresentam padrão de preensão maduro, enquanto somente $42,5 \%$ das meninas apresentam esse padrão. 
Silva CG, et al. Análise psicométrica dos itens da ACOORDEM. Rev Ter Ocup Univ São Paulo. 2017 jan./abr.;28(1):9-18.

Tabela 4 - Descrição e comparação dos itens qualitativos da ACOORDEM - por sexo e tipo de escola

\begin{tabular}{|c|c|c|c|c|c|c|c|c|c|c|}
\hline \multirow{3}{*}{$\begin{array}{l}\text { Itens qualitativos } \\
\text { da ACOORDEM }\end{array}$} & \multicolumn{5}{|c|}{ Sexo } & \multicolumn{5}{|c|}{ Tipo de escola } \\
\hline & \multicolumn{2}{|c|}{ Feminino } & \multicolumn{2}{|c|}{ Masculino } & \multirow{2}{*}{$\begin{array}{c}\text { Valor } \\
\mathbf{p}\end{array}$} & \multicolumn{2}{|c|}{ Particular } & \multicolumn{2}{|c|}{ Pública } & \multirow{2}{*}{$\begin{array}{c}\text { Valor } \\
\mathbf{p}\end{array}$} \\
\hline & $\begin{array}{c}\text { Média } \\
\text { (D.P) }\end{array}$ & Mediana & $\begin{array}{l}\text { Média } \\
\text { (D.P) }\end{array}$ & Mediana & & $\begin{array}{c}\text { Média } \\
\text { (D.P) }\end{array}$ & Mediana & $\begin{array}{l}\text { Média } \\
\text { (D.P) }\end{array}$ & Mediana & \\
\hline \multicolumn{11}{|c|}{ Coordenação Manual e Destreza Motora } \\
\hline $\begin{array}{l}\text { Cópia de figuras- } \\
\text { quadrado } \varnothing\end{array}$ & $0,80(0,41)$ & 1,0 & $0,72(0,46)$ & 1,0 & 0,397 & $0,78(0,42)$ & 1,0 & $0,74(0,44)$ & 1,0 & 0,745 \\
\hline Cópia de figuras- $\mathrm{X} \not$ & $0,13(0,33)$ & 0,0 & $0,30(0,46)$ & 0,0 & 0,057 & $0,25(0,44)$ & 0,0 & $0,18(0,38)$ & 0,0 & 0,415 \\
\hline Cópia de figuras- peixe $\varnothing$ & $0,23(0,42)$ & 0,0 & $0,05(0,22)$ & 0,0 & 0,024 & $0,18(0,38)$ & 0,0 & $0,10(0,30)$ & 0,0 & 0,333 \\
\hline $\begin{array}{l}\text { Cópia de figuras- } 3 \text { linhas } \\
\text { cruzadas } \varnothing\end{array}$ & $0,00(0,0)$ & 0,0 & $0,03(0,16)$ & 0,0 & 0,317 & $0,03(0,15)$ & 0,0 & $0,00(0,00)$ & 0,0 & 0,317 \\
\hline $\begin{array}{l}\text { Cópia de figuras- círculo/ } \\
\text { quadrado aberto } \varnothing\end{array}$ & $0,28(0,45)$ & 0,0 & $0,23(0,42)$ & 0,0 & 0,608 & $0,33(0,47)$ & 0,0 & $0,18(0,38)$ & 0,0 & 0,124 \\
\hline $\begin{array}{l}\text { Cópia de figuras- } 3 \\
\text { círculos } \varnothing \\
\end{array}$ & $0,10(0,30)$ & 0,0 & $0,15(0,36)$ & 0,0 & 0,502 & $0,18(0,38)$ & 0,0 & $0,08(0,27)$ & 0,0 & 0,179 \\
\hline $\begin{array}{l}\text { Cópia de figuras- círculo } \\
\text { e triângulo } \varnothing\end{array}$ & $0,03(0,16)$ & 0,0 & $0,08(0,27)$ & 0,0 & 0,308 & $0,08(0,27)$ & 0,0 & $0,03(0,16)$ & 0,0 & 0,308 \\
\hline $\begin{array}{l}\text { Cópia de figuras- losango } \\
\text { horizontal } \varnothing\end{array}$ & $0,03(0,16)$ & 0,0 & $0,00(0,0)$ & 0,0 & 0,317 & $0,03(1,34)$ & 2,0 & $0,00(0,0)$ & 0,0 & 0,317 \\
\hline \multicolumn{11}{|c|}{ Coordenação Bilateral e planejamento motor } \\
\hline Batucada $1 \varnothing$ & $2,03(0,89)$ & 2,0 & $2,25(0,87)$ & 3,0 & 0,251 & $2,33(0,83)$ & 3,0 & $1,95(0,90)$ & 2,0 & 0,061 \\
\hline Batucada $2 \varnothing$ & $2,15(0,97)$ & 3,0 & $2,20(0,91)$ & 3,0 & 0,871 & $2,33(0,88)$ & 3,0 & $2,03(0,97)$ & 2,0 & 0,166 \\
\hline Batucada $3 \varnothing$ & $1,40(0,77)$ & 1,0 & $1,33(0,62)$ & 1,0 & 0,985 & $1,53(0,78)$ & 1,0 & $1,20(0,56)$ & 1,0 & 0,022 \\
\hline Batucada $4 \varnothing$ & $1,97(0,98)$ & 2,0 & $1,95(0,99)$ & 1,5 & 0,912 & $2,30(0,94)$ & 3,0 & $1,62(0,90)$ & 1,0 & 0,002 \\
\hline $\begin{array}{l}\text { Amarelinha } 2 \text { pés } \\
\text { laterais } \varnothing\end{array}$ & $2,30(0,64)$ & 2,0 & $2,38(0,70)$ & 2,5 & 0,525 & $2,45(0,67)$ & 3,0 & $2,23(0,66)$ & 2,0 & 0,105 \\
\hline Amarelinha $1221 \varnothing$ & $1,78(0,80)$ & 2,0 & $1,80(0,82)$ & 2,0 & 0,909 & $2,00(0,85)$ & 2,0 & $1,58(0,72)$ & 1,0 & 0,022 \\
\hline Amarelinha $12112 \varnothing$ & $1,83(0,78)$ & 2,0 & $1,68(0,83)$ & 1,0 & 0,333 & $1,98(0,83)$ & 2,0 & $1,53(0,72)$ & 1,0 & 0,013 \\
\hline Polichinelo $\varnothing$ & $1,25(0,54)$ & 1,0 & $1,30(0,61)$ & 1,0 & 0,111 & $1,30(0,61)$ & 1,0 & $1,05(0,22)$ & 1,0 & 0,021 \\
\hline Prono extensão acuidade & $1,88(0,68)$ & 2,0 & $1,78(0,66)$ & 2,0 & 0,348 & $1,78(0,66)$ & 2,0 & $1,83(0,67)$ & 2,0 & 0,790 \\
\hline Supino flexão acuidade $\varnothing$ & $2,14(0,85)$ & 2,0 & $2,08(0,86)$ & 2,0 & 0,304 & $2,08(0,86)$ & 2,0 & $1,97(0,69)$ & 2,0 & 0,688 \\
\hline
\end{tabular}

\section{DISCUSS ÃO}

$\mathrm{Na}$ aplicação da ACOORDEM com crianças de 4 anos, o teste manteve a qualidade revelada nos estudos feitos com crianças de 6 a 8 anos de idade. $O$ teste foi bem aceito pelas crianças, muitos itens apresentam índices aceitáveis de confiabilidade teste reteste e foi identificada pouca influência do sexo e tipo de escola no desempenho motor.
Considerando os estudos prévios da ACOORDEM $^{12,13}$ quando comparado o desempenho motor por sexo, em crianças mais novas, observase diminuição dos itens com diferença estatística significante. Aos 7 e 8 anos foram observadas diferenças em 11 itens $^{12}$, e aos 6 anos em nove itens ${ }^{13}$. Neste estudo somente três itens apresentaram diferença estatística significante. Crianças mais novas estão adquirindo habilidades motoras e, portanto, é difícil diferenciar o 
comportamento motor pelo sexo. Em um estudo sobre as percepções de competência e desenvolvimento motor Valentini ${ }^{19}$ também não encontrou diferença significativa no desempenho motor de meninos e meninas de 5 a 10 anos. Contudo meninos evidenciaram superioridade nas habilidades de controle de objetos. Essa diferenciação motora é maior a partir dos 6 anos, quando meninos passam a sobressair em tarefas motoras que necessitam de potência muscular, enquanto as meninas são melhores em tarefas que necessitam de equilíbrio e flexibilidade ${ }^{20}$. Assim, os achados deste estudo corroboram com os estudos anteriores de validação da ACOORDEM que indicaram não haver necessidade de criação de tabelas normativas diferenciadas por sexo.

Na comparação do desempenho motor por tipo de escola também foi observada redução do número de itens com diferença estatisticamente significante quando comparado aos estudos prévios da ACOORDEM. Entretanto, o desempenho das crianças das escolas particulares se manteve superior ao das escolas públicas, como observado nos estudos anteriores da ACOORDEM ${ }^{12,13}$. Fatores ambientais podem ter influenciado esse resultado, pois foi observado que nas escolas particulares havia maior oferta de atividades motoras diferenciadas, como balé, natação, basquete e futebol. Santos et al. ${ }^{21}$ ao investigar as habilidades cognitiva e motoras fina e grossa de pré-escolares matriculados em creches públicas e particulares do interior de São Paulo, também evidenciaram diferença estatística significante para as três habilidades investigadas, com crianças de escolas particulares obtendo desempenho superior. Essa diferença no comportamento motor foi atribuída ao estímulo ambiental na escola e em casa, uma vez que a estrutura física e possibilidades de estimulação da criança de escolas particulares foram maiores. Barbosa et al. ${ }^{22}$ descrevem que o recreio é o principal momento de oferta de atividades físicas em escolas municipais, já que não há outras fontes de atividades físicas como atividades esportivas e extra curriculares. Apesar das diferenças ambientais, é importante ressaltar que não há relação direta entre o nível sócio econômico e bom desempenho motor, uma vez que nem sempre crianças de classe social mais alta apresentam melhor desempenho por ter acesso a diferentes atividades e recursos ${ }^{23}$. Assim, mais estudos serão necessários para esclarecer a relação entre o nível sócio econômico, fatores ambientais e o desempenho motor.

No que diz respeito ao padrão de preensão no lápis, foi observado que grande parte das crianças avaliadas $(48,8 \%)$ apresentou padrões de preensão maduros, sendo a preensão mais frequente a trípode lateral (27,5\%), seguido da trípode dinâmica $(21,3 \%)$. Esse é um dado interessante, pois nessa idade são esperados padrões de transição ${ }^{14}$, como a preensão de 4 dedos que, neste estudo foi observado em 28,8\% das crianças. Esse dado é sugestivo de que crianças brasileiras estão sendo estimuladas a usar o lápis mais precocemente, o que precisa ser melhor investigado. $\mathrm{Na}$ literatura, a prevalência da trípode dinâmica, padrão considerado ergonomicamente mais estável, varia de 33 a $67 \%$ em crianças acima de 7 anos de idade e adultos ${ }^{24}$.

Considerando o reteste a análise dos itens quantitativos da ACOORDEM indica estabilidade temporal, já que $78 \%$ dos itens apresentaram boa consistência. O mesmo ocorreu com os itens qualitativos, dos quais $94,4 \%$ apresentaram confiabilidade teste reteste de moderada a excelente. Nos estudos anteriores a confiabilidade teste reteste dos itens de equilíbrio corporal e coordenação bilateral/sequenciamento motor foram investigados em crianças de 4,6 e 8 anos, obtendo-se índices de 0,80 em $62,5 \%$ dos itens e 0,60 a 0,79 em $35 \%$ dos itens; e acima de 0,60 em somente $45,7 \%$ dos itens, respectivamente. Resultado similar ao optido aos 6 anos $^{13}$ quando somente $40 \%$ dos itens de coordenação bilateral e planejamento motor obtiveram CCI bom a excelente ${ }^{9,13}$.

Observa-se, no presente estudo, que a confiabilidade teste reteste dos itens quantitativos foi mais alta provavelmente devido ao uso do teste $t$-student pareado, uma vez que ele não exige concordância absoluta dos itens, como o CCI. Em estudos futuros os dados brutos devem ser transformados em escores padronizados, para serem submetidos a CCI. Apesar dos índices aceitáveis encontrados, o tempo de aplicação do teste e a complexidade de alguns itens são pontos que ainda precisam ser revistos.

Considerando a extensão do teste, o tempo de aplicação da ACOORDEM é longo para crianças de 4 anos, uma vez que as crianças nessa idade tendem a ser pouco colaborativas. Além disso, foi necessário que a avaliação ocorresse em dupla para estimular o engajamento nas provas do teste durante todo o período de aplicação.

Foram também detectados itens com complexidade alta para a idade de 4 anos, como por exemplo "cópias de figuras". Amundson ${ }^{25}$ relata que crianças de 4 anos são capazes de desenhar linhas horizontais, verticais, diagonais, cruzes, círculos e quadrados, habilidade que já é exigida em uma das 
provas de escrita, na qual as crianças reproduzem esses símbolos. Entretanto, o item "cópia de figuras" solicita a reprodução de figuras mais elaboradas, que resultou em baixo desempenho da amostra examinada por ser uma exigência superior à esperada para a idade. É importante, assim, definir critério de interrupção, para evitar que crianças de 4 e 5 anos façam itens para os quais não têm habilidade. Outros testes como Developmental Test of Visual-Motor Integration (VMI) interrompem a prova após três erros consecutivos. Outros itens como "Polichinelo", "Repicar bola 20 cm", "Atirar bola na parede", "Supino flexão - tempo" e "Prono extensão - tempo" obtiveram escores muito baixos, o que também indica complexidade elevada. Deve-se, portanto, analisar cuidadosamente o padrão de escores de cada item para verificar sua adequação para crianças de 4 anos. Um teste mais curto facilitaria o manejo clínico da avaliação.

Com relação às limitações do estudo, as escolas que compuseram a amostra foram recrutadas por conveniência, não constituindo necessariamente uma amostra representativa do universo escolar do país.
Porém as crianças foram recrutadas por meio de sorteio, para garantir igual chance de recrutamento em cada turma das escolas participantes.

\section{CONCLUSÃO}

A ACOORDEM demonstrou ser um teste motor válido para avaliação da coordenação e destreza motoras das crianças brasileiras de 4 anos de idade. Foram encontradas poucas diferenças significativas no desempenho dos itens da ACOORDEM ao comparar sexo e tipo de escola, o que sugere que o processo de normatização do teste pode seguir com tabela normativa única, sem necessidade de diferenciação por sexo ou tipo de escola, o que deve ser confirmado com a amostra total de validação do teste. A confiabilidade teste reteste dos itens da ACOORDEM foi satisfatória, com a maioria apresentando bons índices de confiabilidade.

Foram evidenciadas limitações na ACOORDEM, sendo recomendada adequação da complexidade dos itens e redução do tempo de aplicação do teste para crianças pré-escolares.

Agradecimentos: Agradecimento às instituições, professores, famílias e crianças que participaram deste estudo. Projeto de pesquisa financiado pelo Conselho Nacional de Desenvolvimento Científico e Tecnológico (CNPq) e Fundação de Amparo à Pesquisa do Estado de Minas Gerais (FAPEMIG).

\section{REFERÊNCIAS}

1. Piek JP, Hands B, Licari MK. Assessment of motor functioning in the preschool period. Neuropsychol Rev. 2012;22(4):402-13. http://dx.doi.org/10.1007/s11065-0129211-4.

2. GottliebG.Therelevanceofdevelopmental-psychobiological metatheory to developmental neuropsychology. Dev Neuropsychol. 2001;19(10):1-9. http://dx.doi.org/10.1207/ S15326942DN1901_1.

3. Linde BW, Netten JJ, Otten E, Postema K, Geuze RH, Schoemaker MM. A systematic review of instruments for assessment of capacity in activities of daily living in children with developmental co-ordination disorder. Child Care Health Dev. 2015;41(1):23-34. http://dx.doi.org/10.1111/ cch.12124.

4. Valentini NC, Ramalho MH, Oliveira MA. Movement Assessment Battery for Children-2: Translation, reliability, and validity for Brazilian children. Res Dev
Disabil. 2014;35(3):733-40. http://dx.doi.org/10.1016/j. ridd.2013.10.028.

5. American Psychiatric Association. Manual diagnóstico e estatístico de transtornos mentais: DSM-V. 5a ed. Porto Alegre: Artmed; 2013.

6. Prunty MM, Barnett AL, Wilmut K, Plumb MS. Handwriting speed in children with developmental coordination disorder: are they really slower? Res Dev Disabil. 2013;34(9):292736. http://dx.doi.org/10.1016/j.ridd.2013.06.005.

7. Graham S, Weintraub N. A review of handwriting research: Progress and prospects from 1980 to 1994. Educ Psychol Rev. 1996;8:7-87. http://dx.doi.org/10.1007/BF01761831.

8. Magalhães LC, Nascimento VCS, Rezende MB. Avaliação da coordenação e destreza motora- ACOORDEM: etapas de criação e perspectivas de validação. Rev Ter Ocup Univ 
São Paulo. 2004;15(1):17-25. http://dx.doi.org/10.11606/ issn.2238-6149.v15i1p17-25.

9. Cardoso AA, Magalhães LC. Bilateral coordination and motor sequencing in Brazilian children: preliminary construct validity and reliability analysis. Occup Ther Int. 2009;16(2):107-21. http://dx.doi.org/10.1002/oti.273.

10. Cardoso AA, Magalhães LC, Lacerda TTB, Andrade PMO. Relação entre a Avaliação da Coordenação e Destreza Motora (Acoordem) e a Classificação Internacional de Funcionalidade, Incapacidade e Saúde (CIF). Fisioter Mov. 2012;25(1):31-4. http://dx.doi.org/10.1590/S010351502012000100004 .

11. Cury RLM, Magalhães LC. Criação de um protocolo de avaliação do equilíbrio corporal em crianças de quatro, seis e oito anos de idade: uma perspectiva funcional. Rev Bras Fisioter. 2006;10:346-53. http://dx.doi.org/10.1590/S141335552006000300015 .

12. Cardoso AA, Magalhães LC. Análise da validade de critério da avaliação da coordenação e destreza motora ACOORDEM para crianças de 7 e 8 anos de idade. Rev Bras Fisioter. 2011;16:16-22. http://dx.doi.org/10.1590/ S1413-35552012000100004.

13. Agostini OS, Magalhães LC, Campos AF. Assessment of motor coordination and dexterity of six years old children: a psychometric analysis. Motriz Rev Educ Fís. 2014;20(2):16776. http://dx.doi.org/10.1590/S1980-65742014000200006.

14. Schneck C M, Henderson A. Descriptive analysis of the developmental progression of grip position for pencil and crayon control in nondysfunctional children. Am J Occup Ther. 1990;44(10):893-900. http://dx.doi.org/10.5014/ ajot.44.10.893.

15. Schwellnus H, Carnahan H, Kushki A, Missiuna C, Polatajko $\mathrm{H}$, et al. Effect of pencil grasp on the speed and legibility of handwriting in children. Am J Occup Ther. 2012; 66:718726. http://dx.doi.org/10.5014/ajot.2012.004515

16. Schneck CM. Comparison of pencil-grip patterns in first graders with good and poor writing skills. Am J Occup Ther. 1991;45(8):701-6. http://dx.doi.org/10.5014/ajot.45.8.701.
17. Alves ICB, Duarte JLM. Padronização Brasileira da Escala de Maturidade Mental Colúmbia. In: Burgemeister BB, Blum LH, Lorge I. Escala de Maturidade Mental Colúmbia - Manual para Aplicação e Interpretação. 3th ed. São Paulo: Casa do Psicólogo; 1993.

18. Cicchetti DV. Guidelines, criteria, and rules of thumb for evaluating normed and standardized assessment instruments in psychology. Psychol Assess. 1994;6(4):284. http://dx.doi. org/10.1037/1040-3590.6.4.284.

19. Valentini NC. Percepções de competência e desenvolvimento motor de meninos e meninas: um estudo transversal. Movimento. 2002;8(2):51-62.

20. Malina RM, Bouchard C, Bor-Or O. Growth, maturation, and physical activity. Champaign Ill.: Human Kinetics; 2004. http://dx.doi.org/10.1249/00005768-199207000-00018.

21. Santos MM, Corsi C, Marques LAP, Rocha NACF. Comparison of motor and cognitive performance of children attending public and private day care centers. Braz J Phys Ther. 2013;17(6):579-87. http://dx.doi.org/10.1590/S141335552012005000126 .

22. Barbosa SC, Coledam DHC, Neto AS, Elias RGM, Oliveira AR. Ambiente escolar, comportamento sedentário e atividade física em pré-escolares. Rev Paul Pediatr. 2016;34(3):301-8. http://dx.doi.org/10.1016/j.rpped.2016.01.001.

23. Nascimento Junior JRA, Ferreira L, Vissoci JRN, da Silva, PN, Caruzzo NM, Vieira JLL. Nível socioeconômico e affordances do ambiente domiciliar: implicações para o desempenho motor infantil. Rev Edu Fís/UEM. 2015;25(4):651-62. http:// dx.doi.org/10.4025/reveducfis.v25i4.26529.

24. Koziatek SM, Powell NJ. Pencil grips, legibility, and speed of fourth-graders' writing in cursive. Am J Occup Ther. 2003;57:284-8. http://dx.doi.org/10.5014/ajot.57.3.284.

25. Amundson SJ. Prewriting and handwriting skills. In: CaseSmith J. Occupational therapy for children. 5th ed. St Louis, MO: Mosby Elsevier; 2005. p.587-614. 